\title{
MUSCLE ARCHITECTURE IN RELATION TO FUNCTION
}

\author{
Carl Gans* and Aggot S. Gaunt \\ "Department of Biology, 2127 Kraus Natural Science Building. The University of Michigan. Ann Arbor, Michigan 48109 \\ and tDepartment of Zoology. Ohio State University, Columbus, Ohio 43210 U.S.A.
}

\begin{abstract}
Animal muscles generate forces and induce movements at desirable rates. These roles are interactive and must be considered together. Performance of the organism and survival of the species also involve potential optimization of control and of energy consumption. Further, individual variability arising partly via ontogeny and partly from phylogenetic history often has pronounced and sometime conflicting effects on structures and their uses. Hence. apimal bodies are generally adequate for their tasks rather than being elegantly matched to them. For muscle, matching to role is reflected at all levels of muscular organization, from the nature of the sarcoplasm and contractile filaments to architectural arrangernents of the parts and whole of organs.

Vertebrate muscles are often analyzed by mapping their placement and then "explaining" this on the besis of currently observed roles. A recent alternative asks the obverse; given a mass of tissue that may be developed and maintained at a particular cost, what predictions do physical principles permit about its placement. Three anchitectural pattems that deserve discussion are the classical arrangement of fibers in pinnate patterns, the more recent assumption of sarcomere equivalence, and the issue of compartmentation. All have potential functional implications.

1. The assumption of equivalence of the sarcomeres of motor units allows predictions of the fiber length between sites of origin and insertion. In musculoskeletal systems that induce rotation, the observed (but not the pinnationassociated) insertion angle will differ with the radial lines on which the fibers inser. In a dynamic contraction inducing rotation, a shift of moment arm has no effect for muscles of equal muss.

2. Classical pinnate muscles contain many relatively short fibers positioned in parallel but at an angle to the whole muscle, reducing the per fiber force contribution. However, the total physiological cross-section and total muscle force ane thus increased relative 10 arrangements with fibers parallel to the whole muscle. Equivalent muscles may be placed in various volumetric configurations matching other demands of the organism. The loss of fiber force due to (pinnate, not equivalent) angulation is compensated for by the reduced shortening of fibers in multipinnate arrays.

3. Comparmentation, i.e.. the subdivision of muscles into independently controlled, spatially discrete volumes. is likely ubiquitous. Differential activation of the columns of radial arrays may fecilitate change of vector and with this of function. Compartmentation is apt to be particularly important in strap muscles with shon fiber architecture; their motor units generally occupy columnar, rather than transversely stacked, subdivisions; this may affect recovery from fiber atrophy and degeneration. Transverse and columnar stacking occur; both may have historical, as well as functional causes.
\end{abstract}

\section{INTRODUCTION}

The nature of muscles and their placement have been subject to past and continuing selection, presumably leading to the current matching to their environment referred to as adaptation. Adaptation of muscles may have modified them for one or a combination of multiple roles, such as (1) effectiveness (efficiency) in terms of energy consumption, (2) generation of short-term "maximum" force, or (3) continuous low level force (Gans, 1991). Adaptation may have affected other aspects of performance, such as neuronal control of the muscle activation to maintain position and effective muscle output.

However, any individual needs only to be adequate for the demands encountered during its lifetime! Perfection and optimization are abstract concepts, rather than conditions likely to be encountered in real organisms. In the present context this caveat is important because any structural system incorporates diversity, sometimes due to history, either ontogenetic from embryo to adult, or phylogenetic covering evolutionary time. Hence, we need not expect that all of the observed individual conditions and all aspects of transitional stages will beautifully reflect functional demands. They need only be sufficient for the environmental states then encountered.

Also, there is physiological adaptation (Prosser, 1958), another usage of the term, referring to the capacity of some tissues to produce useful changes to signals deriving from an altered environment. Although this capacity is sometimes assumed to represent a purely phenotypic effect, this ontogenetic response is, in fact, genetically based. It does not differ from other ontogenetic aspects, as any phenotype always derives both from genetic instructions and environmental influences. The capacity to respond at a particular level of physiological adaptation reflects natural selection.

In evolutionary srudy, adaptations are generally approached by examining existing structural and physiological conditions and establishing the way that they facilitate survival. Much of what is now referred to as functional morphology consists of describing the structure and then considering its function. How does the structure match its role, namely what 2spects are now under the influence of natural selection? In such studies, the questions become: 
Does a cat's jaw let it bite, and how hard will the bite be? Which aspects of the legs of a horse permit it to run; what kinds of nunning occur and how do these match aspects of structure and environmental demands? Can birds fly and how fast, straight, high and long do they do so? How good is the performance in each case? Too often the answers imply that the conditions seen are "necessary" for current survival, even in the absence of direct evidence. For instance, $L$ : individual details of any muscular arrangement are assumed to represent states that must be explained by current needs of the particular organism (Ruben, 1991). The best such studies in functional morphology have shifted to the field and ask how particular characters are associated with the animal's ultimate task, that of passing its genes to the next generation (e.g., Feder and Huey. 1987; Huey and Dunham, 1987).

An alternative approach to analysis is synthetic. It starts by studying the properties of muscle, tendons, aponeuroses and bones. From these it asks about the relative merits of installing a particular quantity of such tissues in order to perform the role. The questions then become: Given a gram of muscle, where should this tissue be placed to achieve the greatest benefit for the cost that it imposes on the organism? What is the effect of slightly different placements? How should benefit be defined: in terms of excursion, of force generation, of work, or of power? How would the answer be affected if the mass of muscle were doubled? What would be the effect of modification of the connective and skeletal tissues? Comparison of the answers leads to a test of the relative importance of functional and historical causes in explaining the observed diversity.

This account will deal with architecture, namely the placement of sarcomeres in fibers, with the fibers in muscles, and with the muscles in animals; this is an intermediate level of analysis that is often ignored. Both displacement and force will be affected by the way that fibers are attached to each other, to tendons and aponeuroses, and to skeletal elements. What is the effect of such muscle placement or muscle architecture on the function of the component muscles and the performance of the entire animal?

In the present framework it is impossible to review all of the vast literature dealing with the topics of architecture and muscle placement. All of these topics were discovered and periodically rediscovered over some six score years and as regularly ignored or forgotten. Here, we would like to recall attention to the understanding of the intermediate level of architecture. It seems useful to provide at least an introduction and some initial references to the implications of sarcomere equivalence, pinnation and compartmentation within muscles. We hope that this may force a re-evaluation of such currently held concepts as the importance of moment arm, the implication that all fiber angulation is equivalent to pinnation and that single fibers run from origin to insertion of muscles.

Here, we propose to examine three different architectural arrangements of muscle fibers: (1) Equivalence. (2) Pinnation, and (3) Compartmentalization. The force/ shortening trade-off and position of insertion will be considered for each.

\section{OPTIMIZATION}

Selection by an animal of a "desirable" muscle placement (and our theoretical conclusion that such selection has indeed taken place) requires that there exists a standard by which a comparison may be made. Obviously, the muscles empower the animal to perform those actions that provide some match to its environmental circumstances; in evolutionary terms, an animal may invade and survive in any place in which it can make a living. A population will survive in the longer term in any place in which a sufficiency of its members can achieve reproduction. Within such a framework there may be a benefit to more effective muscles, effectiveness being affected by myofiber physiology as well as muscle placement and architecture (Otten, 1988. 1989). However, it is the overall performance and not mere contraction effectiveness that is important to the organism.

Concepts of design in industry and nature imply that a desirable state can be approached to a desired level of precision. This may be possible in the engineering of particular machine components; here, the problem of growth is generally avoided. However, in nature it is not only necessary to function while growing. but organisms are notorious for using single tissues and structures for multiple, often temporally distinct, tasks. It has long been accepted that conflicts among the demands of these tasks keeps the elements from being selected for absolute efficiency in the performance of any one aspect (Gans, 1983): however, there is the holistic concept of optimization that might be defined as "the best under the circumstances likely to be encountered".

In many ways such a concept of optimization is nonoperational, as the values that are likely established by an optimization equation will differ among species and even among features of an organism. Furthermore, the many factors selecting for the form of each feature will differ in their importance. Consequently, optimization of multiple characters will be affected more by some environmental conditions than by others. Hence, the documentation that some features are optimized for a particular state normally reflects the discovery of the relative values for at least two, often competing, selective factors. The same factors operating at the same relative strengths need not apply to additional species.

In the present case this means that we cannot yet predict the kinds of muscle arrangements that we should expect to see; rather the implications of architectural analysis have each to be matched to the circumstances of the particular organisms being studied. Generalizations about muscular detail still must follow description.

\section{MUSCLE ARCHITECTURE AND FUNCTION}

\section{General}

The structure and chemistry of the cellular components of muscles are adapted for performing multiple roles, including locomotion, breathing, feeding. mating, and 
their interactions. Indeed such movement characterizes animals; one may obtain the impression that the study of muscle may reflect purely reductionist thinking. However, major adaptation appears in behavior, the way the tissue is used; organisms often use behavior to avoid limitations due to physical circumstances in the environment (McMahon, 1984; Otten, 1989; Pennycuick, 1989, 1991; Weeks, 1989).

Muscle effectiveness (sometimes called economy, Rall, 1985; or efficiency, see Gans, 1991) is the amount of energy expended by unit force production or unit shortening. We have long recognized that speed of shortening has a cost and that energetic effectiveness reaches a maximum close to the slowest speeds (expressed as $V / V_{\max }$, where $V$ is actual velocity and $\mathrm{V}_{\max }$ is the maximum or unloaded velocity). It was stated long ago (Howell, 1944; Close, 1972), that the inertia of body segments increases with absolute size and that larger organisms move their elements more slowly than do smailer ones. Hence, step cycles, footfall patterns and chewing movements are seen to proceed more slowly in large than in small species and often slow down during ontogenetic development. Such relations have recently been confirmed in the movement of intact animals (Rome et al. 1988).

The muscles that generate the rotational action of jointed skeletal elements should then insert close to the fulcrum, thereby limiting the required velocity of shortening at an apparent cost of reducing the moment arm for the forces they produce (Biewener, this issue: Carrier, 1989). However, this seeming conflict will be shown to be nonexistent under the heading of "Equivalence".

As contractive effectiveness is generally stated in energetic terms. it involves the distance (or the amount of shortening) over which the force is exerted. In these terms, muscles have zero efficiency while maintaining a posture. This incorporates a conceptual artifact. The muscles have multiple roles: they must maintain particular positions, but must also, and at other times, shorten to bring the components of the animal into desired positions. Apparently, animals have adapted such muscles to expend energy in the maintenance of a combination of stability and movement. This combination may be as likely to have adaptive significance as would a maximum movement rate or a minimal energy consumption by itself.

As interesting is the contrast between excentric and concentric contraction, or stimulation of muscles while they are lengthening or while being allowed to shorten. The potential for force generation is much greater for lengthening muscles. However, muscles cannot lengthen by themselves. In biological systems such lengthening always occurs by the action of other (antagonistic) muscles. generally at earlier times; even external forces, such as those imposed by gravily, reflect previous muscular action, storing potential energy in the system. Excellent examples occur in lengthening of the forelimb muscles of galloping tetrapods; these are stretched upon contact with the ground and thus recover energy built up by earlier shortening of the hindlimb muscles. Also, in jaw muscle systems the closers may be activated while they are still being stretched by the openers. A bcautiful recent example concerns the flight muscles of birds, and demonstrates that the power and recovery muscies of the wing each become activated slightly before the change in the direction of motion (Dial et al., 1988; Goslow and Dial, 1989).

As the force generated is affected by distance and velocity of the myofiber displacement, the position of the attachment sites will be critical. For some, often long. muscles the tendons of attachment may also be long: however, tendon stiffness will limit tendon stretch, allowing the muscle fibers to remain in the desired portion of the length tension range (Peterson et al., 1982, 1984). Greater stiffness, particularly for relatively short fibers, such as sometimes seen in pinnate arrays, seems to be reflected by tendon ossification.

\section{Equivalence}

The sarcomeres comprising the contractile aspect of myofibrils obviously lie in series. Whenever a string of sarcomeres is stimulated, temporal differences of activation and slight differences of resistance generate the potential for slight differences in deformation (Hill, 1970). Similar slight differences are apparently produced by the differential placement of muscle fibers within a muscle (indirect measurements by Martin et al., 1988). Also, differential stiffness among the sarcomeres may generate non-equivalent shortening along each fiber (Julian and Morgan, 1979; Morgan, 1990). The implications of such perturbations may be evaluated by starting with the initial assumption that all of the sarcomeres of a motor unit are of equivalent size and will shorten equivalently upon stimulation (Tardieu et al., 1981).

Here each muscle fiber is initially modelled on the assumption that all of its sarcomeres are equivalent (have equal numbers of overlapping myofilaments and are exposed to equal shortening). The importance of this initial assumption of equivalence can be seen to be critical if it is remembered that force and displacement and, of course, force and velocity, interact. Consequently, the force generated during activation of a muscle fiber reflects the starting length of each sarcomere, its relative shortening and the rate thereof. In this case, each sarcomere in a string (fibril) of constant cross-sectional area will produce equal force for a given relative change of length of the entire muscle; this force will be that exerted by the string as a whole (Fig. 1). The moment generated by the fibril will be equal to the fibril (sarcomere) force times the distance of the fibril from the fulcrum around which the insertion site is made to rotate. The mass-specific moment of the fibril can be defined as the fibril moment divided by fibril mass, or equivalently (assuming equivalent sarcomeres), dividing by the number of sarcomeres comprising the fibril to obtain a sarcomere-specific moment. The further the particular fibril is placed from the fulcrum, the greater its absolute shortening. Hence, the number of component sarcomeres in series must increase proportionally to maintain the relative shortening (of each fiber and of each sarcomere, hence "equivalent" sarcomere length).

In such a model, the moment produced per sarcomere (sarcomere-specific moment) remains constant. For a given 


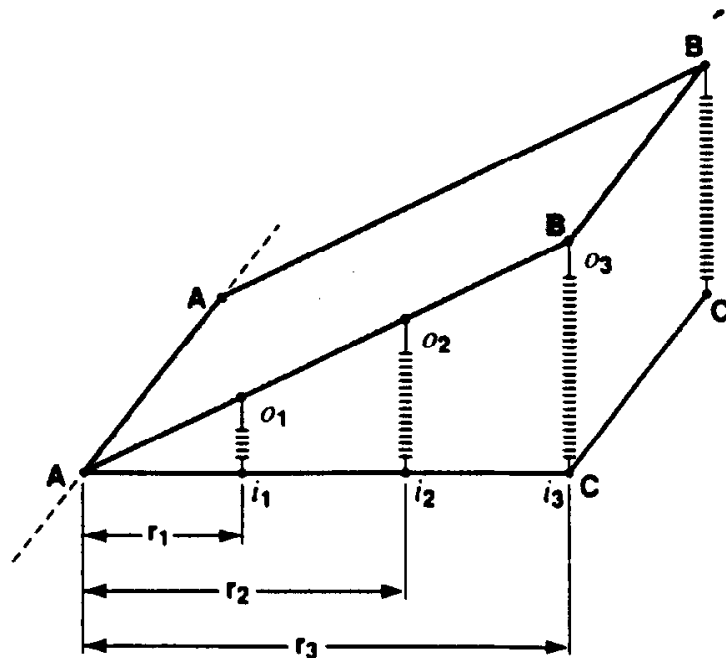

Fig. 1. Wedge of fibers tending to move one surface (AABB) toward another (AACC) by rotational action about axis A-A. The number of sarcomeres of each fiber, placed in paraliel in such a wedge, is likely to be proportional to the length of the fiber $\left(\overline{i_{1} O_{1}}, \overline{i_{2} O_{2}}, \overline{i_{3} O_{3}}\right)$ and hence to the moment arm $\left(\eta, \eta_{2}, \eta_{3}\right)$. Each fibril (sarcomere). in such a wedge of fibers placed in parailel, will produce an equal force and is likely to produce an equal fibril mass-specific moment (or sarcomere-specific moment) for equivalent shortening and to induce equal shortening for equivalent mass-specific moment. This falsifies the common assumption that it is more advantageous to place a specific mass of muscle more distally from a joint capable of a set angular excursion. (Adapted from Gans and de Vree, 1987.)

mass of muscle, i.e., a given number of equivalent sarcomeres, modifying the placement site of the tendon of insertion closer to or away from the fulcrum will not affect the moment generated (Gans, 1988; Gans and de Vree, 1987; see Fig. 1). Modifying the insertion away from the fulcrum may, however, permit the placement of a more massive muscle; ergo, more equivalent sarcomeres in parallel would increase the moment; also, the moment may be increased by limiting the range of shortening of each sarcomere. Whereas the sarcomeres within the muscle will still be equivalent, the demand on each will have changed.

The equivalence model applies for any constant angle of insertion in the plane of rotation. For any joint position, the angle of insertion is measured between the fiber and the line from insertion site and fulcrum. Thus equivalent fibers inserting on a surface above the bite line, such as the coronoid process of the mandible (or an aponeurosis rising therefrom), may have different actual angles (relative to the skull) for different levels of placement and still be functionally parallel-fibered (Fig. 2).

The number of fibers that can be placed equivalently (in the plane of rotation) at a given site will be limited. In almost all animals, the placement of individual fibers is modified by positioning the fibers at an angle to this plane; this represents the classical attachment called pinnation and is discussed in the next section (Gans and Bock. 1965). Also, placement of fibers in functional parallel (equivalency) may require a fiber angulation (angle of equivalence) in the plane of closing independent of that of pinnation (Figs 2 and 3) (Gans and deVree, 1987). However, the relative shortening of the fibers will differ depending on the angle in each of the two planes. Separation of the angles among fibers requires stereotactic analysis, as pinnation and equivalence angulation each will make a different contribution to the effect produced upon stimulation.

The model may of course be modified to consider the implications of regular non-equivalence or differential stiffness along the fiber string. As interesting are its implications if the medial sarcomeres incorporate more myofilaments than the terminal ones (tapered fibers) and if the connective tissue fibers are similarly differentiafed between muscle origin and insertion.

One interesting speculation is that equivalent action of sarcomeres would imply that the excursion of sarcomeres located on the ends of myofibers (or the entire muscle)

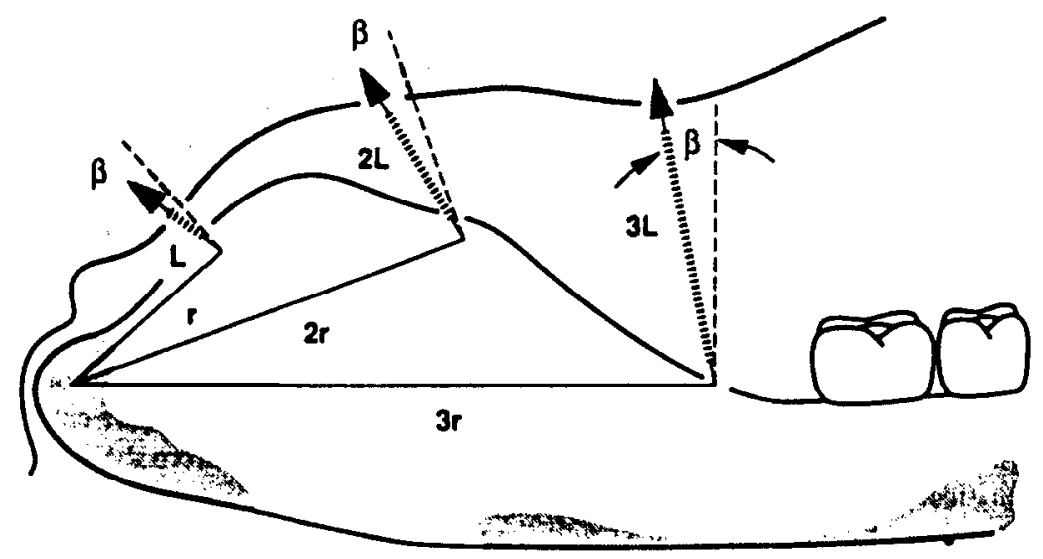

Fig. 2. Diagram illustrating that complex muscles can remain functionally equivalent if the length of each fiber $(L, 2 L, 3 L)$, equivalent to its number of sarcomeres. is maintained proportional to the length of its moment arm $(r, 2 r, 3 r)$. The angle of equivalence, $\beta$, in the plane of closure is illustrated. (Adapted from Gans and de Vree, 1987.) 

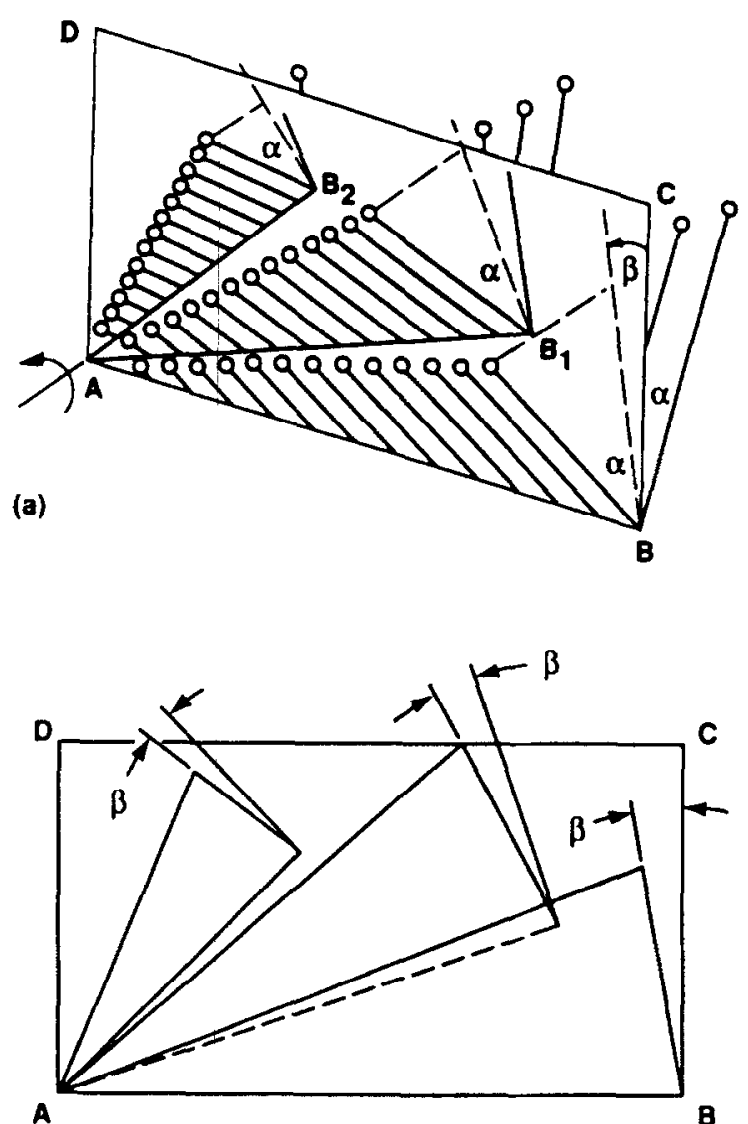

(b)

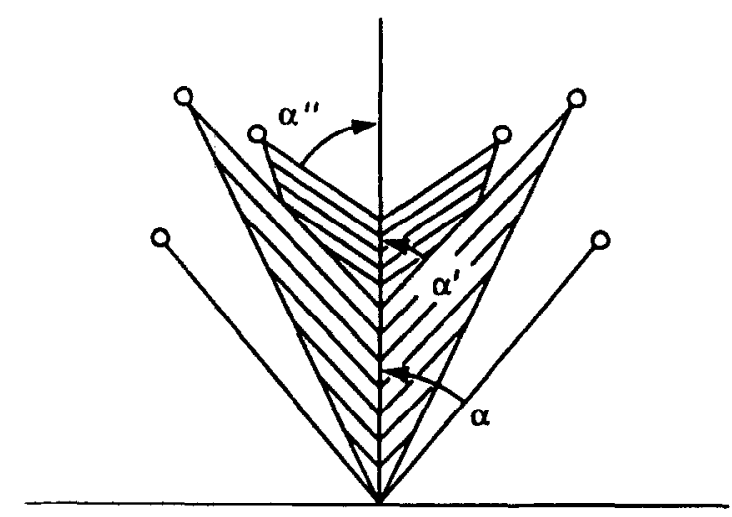

(c)

Fig. 3. A complex muscle can combine pinnation and functionally equivalent placement of sarcomeres. (a) The surface A-B-C-D represents an aponeurosis of a bipinnate muscle rotating about an axis passing normally through it at $A$. The sets of parallel lines represent muscle fibers originating at the small cireles and inserting on the aponeurosis. The solid lines join the insertions of fibers that have parallel moment arms. The angle $\alpha$ is the pinnation angle; it is illustrated as being equivalent through the array. The angle $\beta$ is the angle of equivalence. (b) Lateral view. The angle of equivalence. $\beta$. depends upon the ratio between moment arm ( $A$ $B)$ and the site at which the vector line crosses the performance line. (c) End view. The angle $\alpha$ is the pinnation angle; however, its projection in end view ehanges (c.g.. $\alpha^{\prime}$ and $\alpha^{\prime \prime}$ ). (Adapted from Gans and de Vree, 1985.) would reflect the excursion in the remainder of the fiber (and perhaps the entire muscle). They would contain information that would permit local hypertrophy or atrophy and allow adjustment of the length of the entire muscle in response to changes of the dimensions of the skeletal elements to which it attaches.

\section{Pinnation}

As already noted above. muscle fibers and their fascicles often lie at an angle to the line along which the entire muscle shortens. In some arrays the fibers lying between parallel sheets of connective tissue reverse their direction at each; such arrays are often referred to as pinnate. The minimum definition has been that the line of action of the fibers is not parallel to that of the entire muscle. However, as seen above, equivalence angulation also meets this criterion; hence, a strict definition of pinnation needs the further criterion of having most fibers of the fascicle lying in parallel. The major role of such arrays will, as seen above, be one of packing: thus a given mass of muscle may occupy several equivalent volumetric spaces that will not differ in their length-tension or forcedisplacement relations. In particular, fibers can be arranged along either side of a long tendon (the classic feather-shape) providing a considerable cross-sectional area in a space unsuited to a stout muscle. A subsidiary role resulting from this kind of architecture is that simple pinnate muscles (inserting on aponeuroses that remain parallel during shortening) will not increase diametrically upon stimulation, so that they sometimes occur in bony tunnels ( $M$. tensor tympani) in which binding upon contraction would be detrimental.

The theoretical models of pinnate fibers are drafted with fiber attachment to two parallel bony surfaces that slide past, but do not approach each other. However, many pinnate muscles lie between two unconstrained aponeuroses that show local deformation; this is particularly obvious in singly pinnate arrays, such as the human flexor pollicis longus in which the ends bend as the unilateral contraction induces moments near the attachment site. This produces a localized rise in pressure within the muscular mass (Otten, 1989), although the pressure within muscles arrayed between non-approaching insertion surfaces will not increase (Gans, 1982).

Simple analysis of the excursion of fibers between such non-approaching insertion surfaces, and especially those of multipinnate muscles, discloses that the end of the central tendon moves farther than any myofiber shortens (Fig. 4). This relation used to be considered a minor curiosity of pinnate muscles; however, it may have major functional implications in establishing the force at the tendon. Naturally, only the vector resultant of the force generated by the individual fibers at an angle to the plane of motion will contribute to force at the tendon; thus some force is lost. This loss due to angulation is normally calculated for the static condition, i.e., as a function of the isometric force. However, dynamic experiments and analyses shows that the increased excursion of the central tendon of a pinnate muscle partially compensates for some 


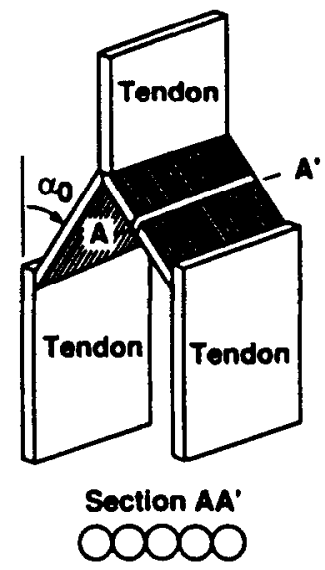

(b)

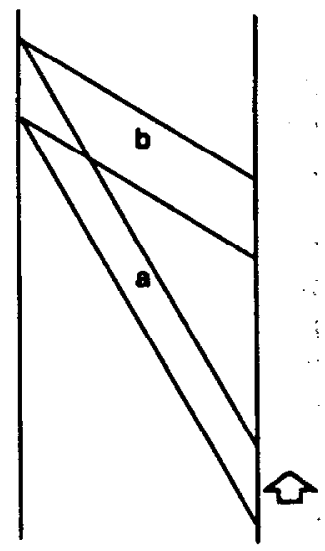

(a)

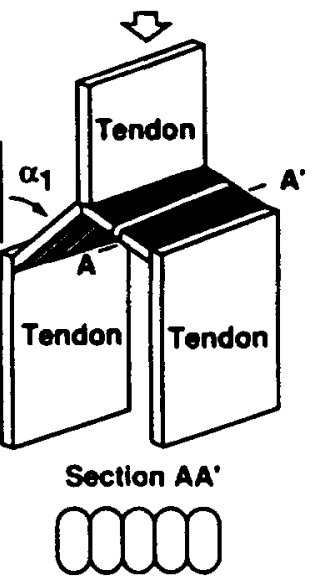

(c)

$$
\begin{aligned}
& \left.\begin{array}{l}
\alpha_{0} \\
L_{0}
\end{array}\right]=\text { Initial } \\
& \left.\begin{array}{l}
\alpha_{1} \\
L_{1}
\end{array}\right]=\text { Final }
\end{aligned}
$$
Fig. 4. Sketches showing a single fiber layer of a loosely placed pinnate muscle and its change with contraction. Assuming that the
surfaces of origin and insertion of the fibers do not approach each other during shortening. the force produced at the tendon will be less than the sum of the forces of the individual fibers. but the displacement of the tendon of insertion will be greater than the absolute shortening of any fiber. Inspection shows that the force of the tendon also varies with the angle and hence changes between start and end of an isotonic shorening sequence. Calculation of the absolute force at the tendon must of course take into account the position of the fiber on the length tension curve and other variables discussed earlier. (b) Sketch showing the change of shape of a contracting fiber in contracting from position " $a$ " to position " $b$." As the areas of the parallelograms are independent of fiber angle, there is no reason to predicate a special build-up of incernal pressure during shortening. However, if the muscle fibers insert on flexible aponeuroses, these will deform and internal pressure will build up. (c) Sketch showing displacements with change of angle. $E_{T}$ is the tendon excursion.

(From Gans, 1982.)

of the loss due to angulation (Muhl, 1982; Gans and de Vree, 1987). Because of the increased excursion, each pinnately arranged sarcomere may shorten over a smaller range of the length tension relation. increasing its mean force output (Fig. 5). The reduced shortening also reduces the absolute contraction velocity required and improves muscle effectiveness.

Pinnation then is a method of packing the required amount of muscle tissue into available sites in the body of an animal. Because the fibers of pinnate muscles act at an angle, their force/velocity relations are not the same as those at the tendon, the displacement of which differs from that of the fibers. The length-tension relationships of the component sarcomeres indicates that the mechanical disadvantage due to the angulation appears to be partially compensated. However, some angled fiber arrangements represent the demands of equivalence and others both equivalence and pinnation, but at right angles to each other. Functional analyses must take the difference into account.

\section{Compartmentation}

Compartmentation, i.e., the subdivision of muscles into independently controlled, spatially discrete volumes, is likely ubiquitous (Barrett, 1962; Peters, 1989). It has been especially well documented, for instance for the lateral gastrocnemius muscle of cats (English and Ledbetter. 1982; English, 1984; English and Weeks, 1984) and also for the masseter of pigs (Herring and Wineski, 1986; 

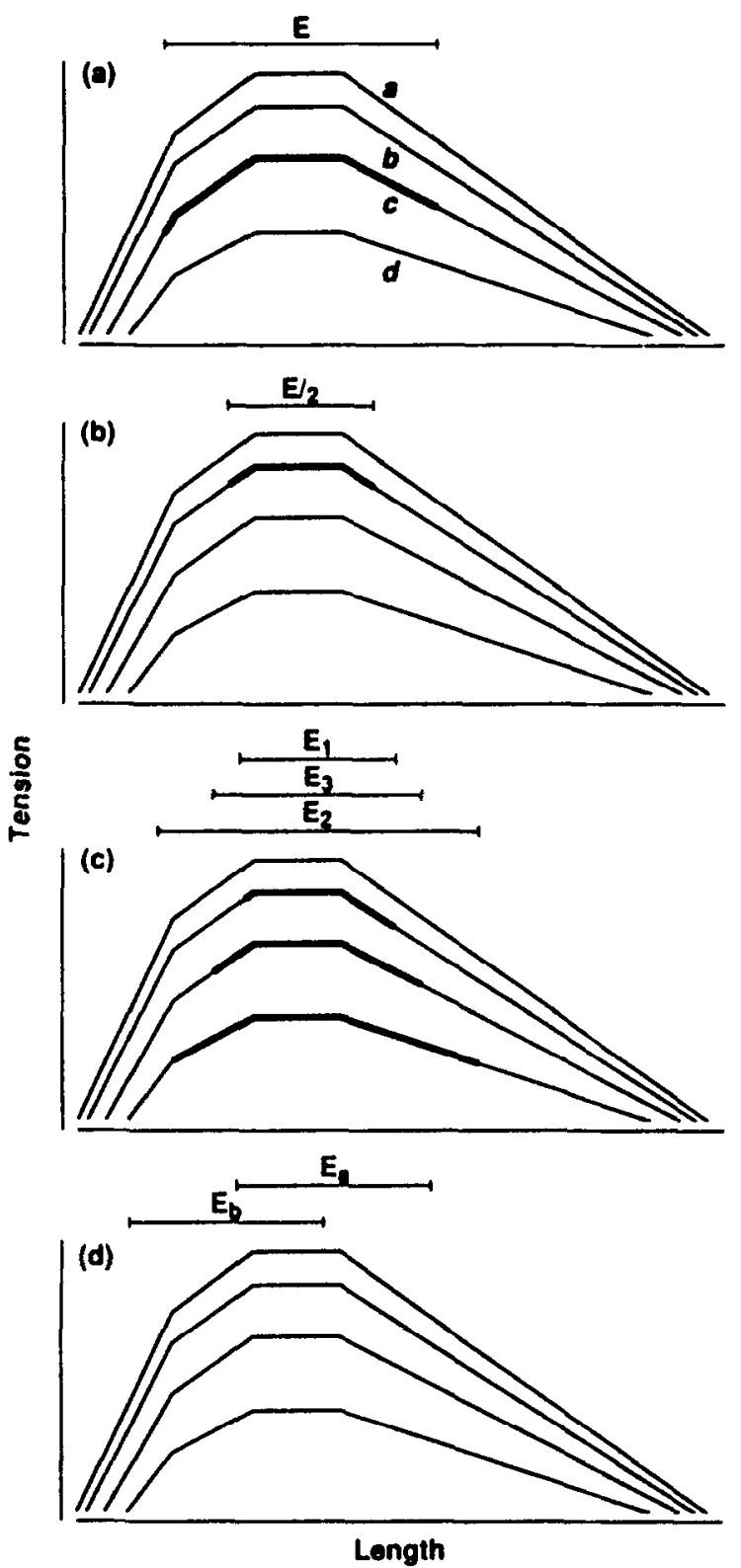

Fig. 5. Length-tension curves of a sarcomere showing its relation to the relative excursion, $E$, imposed on it. (The relative excursion is the excursion of the end of the muscle divided by the number of sarcomeres in series.) In each example, " $a$ " represents an isometric contraction and " $b, c, d$ " show maximal contractions at increasing degrees of shortening velocity. (a) The excursion overlaps the major portion of the range. The shortening velocity proceeds along curve "c." (b) The relative excursion has been reduced $(E / 2)$ by increasing (in this case doubling) the number of sarcomeres in series. This maintains the excursion range closer to the maximum force plateau. Reduction of the excursion required of any sarcomere reduces the velocity, so that the force generated is greater, being mapped along curve " $b$." (c) Effect of pinnation. $E_{1}$ is the relative excursion of a parallel-fibered muscle. As it becomes pinnate, the excursion tends to be increased $10 E_{2}$, because fewer sareomeres are in series. However, the theoretical exeursion value $E_{2}$ is greater than the actual excursion value $E_{3}$, which benefits from the fact that excursion of the central tendon of a bipinnate muscle is greater than the excursion of the individual fibers. (d) Should the maximum demand for force oceur near one end of the excursion range, the sarcomere position can be adjusted so that this excursion correlates with the end of the excursion: $E_{2}$. excursion range adjusted so that the force peaks near the shortest excursion, and $\mathrm{E}_{\mathrm{b}}$ excursion range adjusted so that the force peaks near the longest position. The relative shortening is not affected. (From Gans and de Vree, 1987.)

Wineski and Herring. 1985). Differential activation of the columns of radial arrays, may facilitate change of vector and with this of function (Gaunt and Gans, 1969). However, the biological roles of other kinds of compartmentation are less clearly understood. Here we would like to discuss strap muscles with shor fiber architecture; this latter topic is reviewed here as a special case that is the source of some confusion.

It has recently been noted again that many presumably parallel-fibered strap muscies are rarely comprised of long 
muscle fibers that run from end to end, but commonly of short fibers arranged in overlapping series. This matter is mentioned in numerous textbooks, with the earlier and some recent statements suggesting that the muscle fibers run end to end and some intermediate ones suggesting that the fibers are relatively short and overlapping. Pinnate muscles are commonly supposed to differ from parallelfibered muscles in that the fibers will be shorter. Models including these features may be misleading, however, if they assume that fiber-length relationships are constant and that muscles of primates (including humans) show the same relationships as are found in commonly used experimental species. In fact, all reports of truly long fibers (longer than $6 \mathrm{~cm}$ ) that we have discovered come from human tissue.

The literature is vast and lists numerous examples of long-muscles comprised of short fibers as "exceptions." However, a recent series of studies on cats documents that all long muscles tested show the short fiber architecture and that the ends of the fibers within the muscles are attenuate (tapered), their diameter dropping by a ratio of ten to one with overlaps of some $30 \%$ of the length of individual fibers (Loeb. pers. comm.: Loeb et al., 1987). An anatomical investigation of the semitendinosus muscle of domestic goats showed that these animals also had the short-fiber attenuate-end architecture, and physiological tests also confirm that the short segments are individually innervated (Gans et al., 1989).

When stained, the motor end-plates innervating the succeeding levels of myofibers in a short-fibered muscle form a set of bands along the length of the muscle. We have exploited this phenomenon to sample the semitendinosus muscle from mammals ranging in size from mice to giraffe and hippopotamus, as well as some amphibians and reptiles (Gans and Loeb. pers. obs.). Evidence for short-fiber architecture is found in all taxa. In mammals, certainly, the multi-banding phenomenon is generally size dependent. the smallest species usually showing a single motor endplate band that is usually placed near the middle of each muscle (Fig. 6). Also, data from several mammalian species (cat, goat, pers obs.; cattle, Swatland and Cassens, 1972b; human. Schwarzacher, 1959) indicate that the pattem does not change significantly during ontogeny; thus, the number of myofibers (in series) appears to be constant, and the system extends as it grows.

A parallel project has been proceeding for avian muscle. A key difference (also shared for the frogs, lizards and crocodilians thus far tested) is that avian muscles, no matter how short or from how small a species, show multiple bands of motor end plates. Even some pinnate muscles are multi-banded. Whenever birds are compared to mammals of equivalent size, the avian interband distance is much smaller. An extensive study of several thigh muscles of chickens shows that band numbers and relative interband spacing remains constant both during growth and contraction (Gaunt and Gans, 1990).

The spot-checking on which the above comments are based needs to be tested on a larger sample to establish how widely the short-fiber phenomenon is distributed and to consider possible exceptions. It is interesting that many of the reports of much longer muscle fibers derive from human material, although humans also have some shortfibered muscles (Barret,, 1962; Christensen, 1959; Coârs and Woolf, 1959; Lockhardt and Brandt, 1938; Schwarzacher, 1959). Interestingly, some of the muscles that are short-fibered in humans, e.g., the gracilis, also contain more than one level of cells in mammals as small as mice and rats (Schwarzacher, 1957). The apparent ubiquity of this arrangement in specific muscles suggests that intrafascicularly ending short (and overlapping) myofibers possess some as yet unappreciated property. Further, a possibility exists that some, but definitely not all, of the banding patterns seen reflect multiple innervation of individual fibers (always described for a minority of the fibers of the particular muscle; Feindel et al., 1952; Kordylewski. 1979; Schwarzacher, 1957). Such multiple innervation definitely occurs in all tonic fibers and in most ectotherms (Ashmore et al., 1978: Bamard et al., 1982; Katz and Kuffler, 1941). Not only multiple innervation, but polyneuronal innervation, have been reported for some mammalian muscles (Hunt and Kuffler, 1954; McComas et al. 1984; Sutherland, 1952). The implications of such arrangements for control, development, and function remain unexplored.

Questions remain about the functional and ontogenetic implications of the elongated (tapered) fiber tails. We do not now know whether the spacing between motor endplate bands is a conservative or functionally plastic trait. If the former, whether narrow or wide spacing reflects the ancestral condition; if the latter, the nature of the modifying conditions. Then there are questions about the control of short-fibered strap muscles. In some cases, motor units innervate a column of fibers ranging over multiple levels (Dahm and Landmesser, 1988; Manzano and McComas. 1988; Trotter, 1990), although not necessarily individual fibers arranged in series (Fig. 7). In addition, motor units themselves may be arranged in series and activated differentially (Adrian, 1925; English, 1984; F. C. Richmond, pers. comm.). Such stacking may have historical, as well as functional causes.

Finally, the concatenated short fiber pattern poses problems involved with intermittent loss of fibers due to atrophy and subsequent regeneration, problems that should occur in certain degenerative diseases and prolonged weightlessness (Gans, 1989). Diametric shrinkage of fibers may be reversible by exercise and perhaps by simple stretching (Barnett et al., 1980; McDonagh and Davis, 1984; Gaunt et al., 1990). However, the process assumes that the fibers involved will have remained viable. Furthermore, it incorporates only limited capacity for fiber elongation by the addition of sarcomeres at the fiber ends. Consequently, the loss of all fibers spanning one short level of a muscle may not be reversible without the growth of new fibers between the ends of existing ones. This may explain the persistent muscle weakness of some astronauts (Riley et al. 1990). It also raises questions about which animal models are appropriate for investigating particular questions in muscle physiology (Swatland and Cassens, 1972b). 

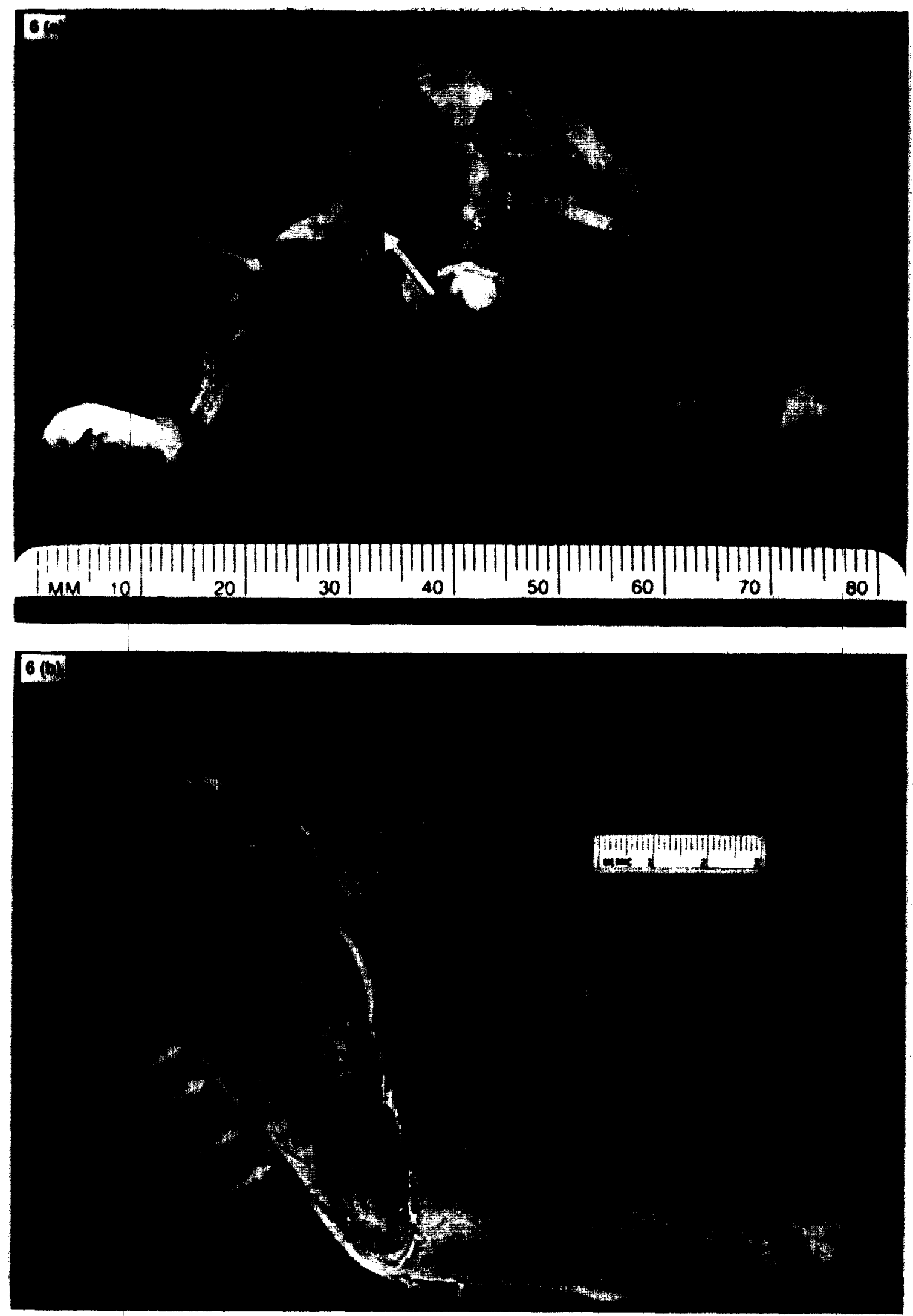

Fig. 6. Top: Mouse, muscles of spread hindquarter stained with acetylcholinesterase (Kamowsky) to show single band of motor endplates at center of several of the muscles (white arrow). Bottom: Hindlimb of newborn goat stained equivalently and showing multiple bands of motor endplates in all muscles (note white arrows for sample bands). 

(a)

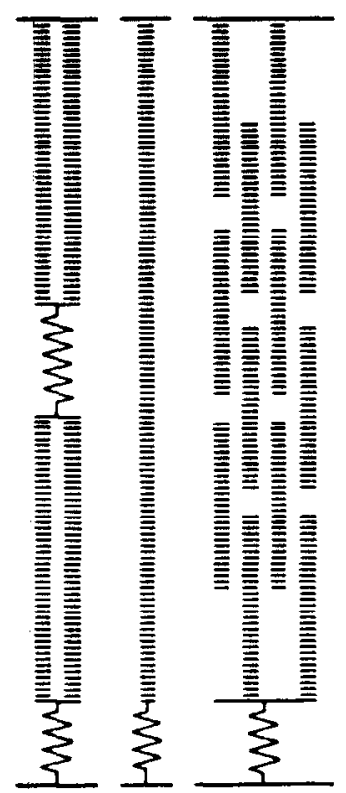

(b)

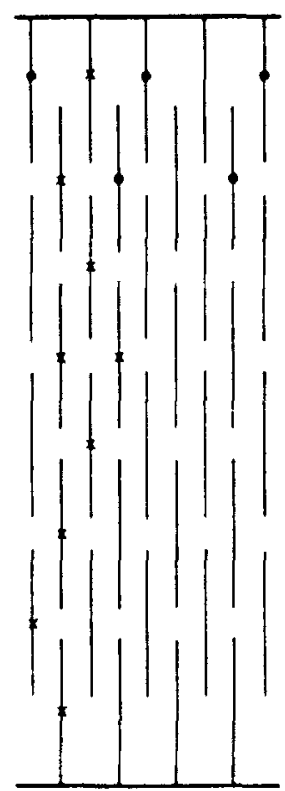

(c)

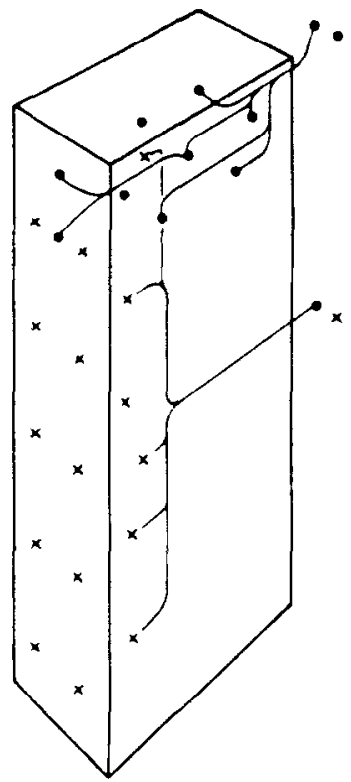

Fig. 7. Schematic arrangement of muscle fibers. (a). Middle: Single fiber connecting two rigid bodies with series elastic connection on one end. (a). Left: Two sets of fibers in series with interealated connective and elastic tissues. (a), Right: Strap muscle composed of short. overlapping fibers. (b) and (c): Two views of short-fibered strap muscles showing organization of motor units transversely, in crossbands (filled circles) and longitudinally, end to end (x's). (From Gans. 1989.)

\section{CONCLUSION}

Analysis of any biological system requires concern about the appropriate levels of structure and organization, the probable interactions among these, and the variation in each. Thus, studies of variation in the structure and placement of muscles (Biewener, this issue) are derivative of studies on the properties of sarcomeres (e.g., Fitts et al., this issue) and of bone (e.g., Martin, this issue); regrettably, they are not ultimately reducible to any one of these, or to simple amalgams thereof. Rather the properties at one level determine the necessity for and extent of trade-offs at another level. The fact that performance is usually subject to competing demands from both intemal (structural and physiological) and extemal (historical and environmental) sources suggests that some optimal (compromise) solution will be selected. Yet, the parameters being selected may vary in number and intensity over time, and the expression of the affected characters will vary among (and possibly within) the individuals comprising any population of animals. Further, any sample of that population will express, and any experimental regime will test, only a fraction of the available variation. Hence, it is scarcely surprising that musculoskeletal models, utilizing optimization of physical or physiological criteria, do not produce clean, precisely accurate results. Yet such studies are absolutely vital for the identification of those elements likely to be essential for a complete analysis and for understanding the interactions among those elements as well as among them and the selecting milieu.
Finally, there is the problem of assessing the meaning of variation in one level for analysis at other levels. Thus, if all the muscles of all species, or of individuals within species, were composed of fibers of similar length that varied according to some simple allometric relationship, then fiber length would be unimportant to functional analyses. However, fiber length varies both among taxa and among the muscles of a species, and this variation shows some regularities. This set of observations makes it probable that fiber length is of some importance per se, at least to some species. However, we are presently unable to say what might be the importance or how it may affect analyses at other levels.

It is hoped that these briefly presented examples have documented that the placement and architecture of muscle fibers represents an important aspect for understanding the biological role of muscles, in other words the useful things they allow the organism to perform. They also establish standards against which actual placements may be tested in order to establish functional meanings. Most important, there remain a number of substantial and as yet unanswered questions considering the intermediate levels of muscle architecture.

Acknowledgements-We thank the organizers of the symposium for the invitation to participate and Dr. R. Whalen for many comments on the manuscript. Preparation supported by a grant from the Leo Leeser Foundation. 


\section{REFERENCES}

Adrian, E. D. (1925) The spread of activity in the tenuissimus muscle of the cat and in other complex muscles. J. Physiol.60, 301-315.

Ashmore, C. R., Kikuchi, T. and Doerr, L. (1978) Some observations on the innervation patuems of different fiber types of chick muscle. Exp. Neurol. 58, 272-284.

Barnard. E. A.. Lyles. J. M. and Pizzey. J. A. (1982) Fibre types in chicken skeletal muscles and their changes in muscular distrophy. J. Physiol. (Lond.) 331, 333-354.

Barnett, J. G., Holly, R. G. and Ashmore, C. R. (1980) Stretchinduced growth in chicken-wing muscles: Biochemical and morphological characterization.Am.J. Physiol.239, C39-C46.

Barrett. B. (1962) The length and mode of termination of indi- vidual muscle fibres in the human sartorius and posterior femoral muscles. Acta Anat. 48, 242-257.

Carner, D. R. (1983) Postnatal ontogeny of the musculo-skeletal system in the black-tailed jackrabbit (Lepus californicus). $J$. Zool. (Lond.) 201, 27-55.

Close. R. I. (1972) Dynamic properties of mammalian skeletal muscles. Physiol. Rev. 52. 129-197.

Christensen, D. (1959) Topography of terminal motor innervations in striated muscles of stillborm infants. Am. J. Phys. Med. 38, 65-78.

Coairs. C. and Woolf, A. L. (1959) The Innervation of Muscle. Blackwell, Oxford.

Dahm, L. M. and Landmesser. L. T. (1988) The regulation of intramuscular nerve branching during normal development and following activity blockade. Dev. Blol. 130. 621-644.

Dial, K. P., Kaplan, S. R., Goslow, G. E., Jr. and Jenkins. F. A., Jr. (1988) A functional analysis of the primary upstroke and downstroke museles in the domestic pigeon (Columba livia) during night. J. Exp. Biol, 134, 1-16.

English, A. W. (1984) An electromyographic analysis of compartments in cat lateral gastrocnemius muscle during unrestrained locomotion. J. Neurophysiol. 52, 114-125.

English, A. W, and Ledbetter, W, D. (1982) A histochemical analysis of identified compartments of cat lateral gastrocnemius muscle. Anat. Rec. 204, 123-130.

English. A. W. and Weeks, O. I. (1984) Comparmentalization of single muscle units in eat lateral gastroenemius. Exp. Brain Res. 56, 361-368.

Feindel, W.. Hinshaw, J. R. and Weddell. G. (1952) The pattern of motor innervation in mammalian striated muscle. J. Anat. 86. 35-47.

Feder, M. and Raymond, E.. Huey, B. (1987) New Directions in Ecological Physiology. Cambridge University Press, Cambridge, New York and Melbourne.

Gans, C. (1982) Fiber architecture and muscle function. Exerc. Sport Sci. Rev. 10. 160-207.

Gans. C. (1983) On the fallacy of perfection. In: Perspectives on Modern Auditory Research: Papers in Honor of E G. Wever. (Edited by Fay, R. R. and Gourevitch, G.). Pp 101-114, Amphora Press, Groton, $\mathrm{Ct}$.

Gans, C. (1988) Muscle insertions do not incur mechanical advantage. Acta Zool. Cracov. 3(25), 615-624.

Gans, C. (1989) Considering functional compartments in muscle. Behavioral Sci. 12(4), 654-655.

Gans. C. (1991) Efficiency, effectiveness, perfection, optimization. Their use in understanding vertebrate evolution. In: Efficiency. Economy and Related Concepts in Comparative Animal Physiology. (Edited by Blake. R. W.). Oxford Univ. Press.

Gans, C. and Bock, W. J. (1965) The functional significance of muscle architecture-a theoretical analysis. In: Ergebnisse der Anatomie und Entwicklungsgeschichte 38, $115-142$. Springer Verlag.

Gans, C. and de Vree. F. (1987) Functional bases of fiber length and angulation in muscle. J. Morphol. 192(1), 63-85.

Gans. C., de Vree, F. and Carrier, D. (1985) Usage pattem of the complex masticatory muscles in the shingle-back lizard. Trachydosaurus rugosus: A model for muscle placement. Am. J. Anat. 173(3), 219-240.

Gans, C., Loeb, G. E. and de Vree, F. (1989) Architecture and physiological properties of the semitendinosus muscle in domestic goats. J. Morphol. 199(3), 287-297.

Gaunt, A. S. and Gans, C. (1969) Mechanics of respiration in the snapping turde. Chelydraserpentina(Linn).J.Morphol. 128(2). 195.228.

Gaunt, A.S. and Gans, C. (1990) Architecture of chicken muscles: Short fiber patterns and their ontogeny. Proc. R. Soc. Lond. 240. 351-362.

Gaunt, A. S., Hikida. R. S., Jehl, J. R., Jr. and Fenbert. L. (1900) Rapid atrophy and hypertrophy of an avian flight muscle. Auk 107. 649-659.

Goslow, G. E., Jr. and Dial, P. K. (1989) Active stretch-shorten contraction of the $M$. pectoralis in the European starling (Sternus vulgaris): Evidence from electromyography and contractile properties. Neth. J. Zool. 40(2), 106-114.

Herring. S. W. and Wineski. L. E. (1986) Development of the masseler muscle and oral behavior in the pig. J. Exp. Zool. 237, 191-207.

Hill, A. V. (1970) First and Last Experiments in Muscle Mechanics. Cambridge University Press, Cambridge, England.

Howell, A. B. (1944) Speed in Animals. Hafner Publ. Co.. New York.

Huey, R. B. and Dunham, A. E. (1987) Repeatability of locomotor performance in natural populations of the lizard Sceloporus merriami. Evol. 41(5). 1116-1120.

Hunt, C. C. and Kuffler, S. W. (1954) Motor innervation of skeletal muscle: Multiple innervation of individual muscle fibres and motor unit function. J. Physiol. (Lond.) 126. 293-303.

Julian, F. J. and Morgan, D. L. (1979) Intersarcomere dynamics during fixed-end tetanic contractions of frog muscle fibres. $J$. Physiol. (Lond.) 293, 365-378.

Katz. B. and Kufner, S. W. (1941) Multiple motor innervation of the frog's sartorius muscle. J. Neurophysiol. 4, 209-223.

Kordylewski. L. (1979) Morphology of muscle fibres in amphibian submandibular muscle. Z Mikrosk. Anat. Forsch. 93(2). 225-243.

Lockhardt, R. D. and Brandt, W. (1938) Length of striated muscle fibers. J. Anat. 72, 470.

Loeb, G. E., Pratt, C. A., Chanaud, C. M. and Richmond, F. J. R. (1987) Distribution and innervation of short. interdigitated muscle fibers in parallel-fibered muscles of the cat hindlimb. J. Morphol. 191.1-15.

Manzano, G. and McComas, A. J. (1988) Longitudinal structure and innervation in two mammalian hindlimb muscles. Muscle and Nerve 11, 115-1122.

Martin, T. P., Bodine-Fowler, S., Roy. R. R., Eldred, E. and Edgerton, V. R. (1988) Metabolic and fiber size properties of cat tibialis anterior motor units. Am. J. Physiol. 255. C43-C50.

McComas, A. J., Kereshi, S. and Manzano, G. (1984) Multiple innervation of human muscle fiber. J. Neurol. Sci. 64, 55-64.

McDonagh, M. J. and Davis, C. T. M. (1984) Adaptive response of mammalian skeletal muscle to exercise with high loads. Eur. J. Appl. Physiol. 52. 139-155.

McMahon. T. A. (1984) Muscles, Reflexes, and Locomotion. Princeton University Press, Princeton, N. J. 
Morgan. D. L. (1990) New insights into the behavior of muscle during active lengthening. Biophys. J. 57. 209-221.

Muhl, 2. F. (1982) Active length-tension relation and the effect of muscle pinnation on fiber lengthening. J. Morphol. 173. 285-292.

Otten. E. (1988) Concepts and models of functional architecture in skeletal muscle. Exerc. Sport Sci. Rev. 16, 89-137.

Otten. E. (1989) Mathematical and philosophical reflections on motor control systems. Acta Morphol. Neerl.-Scand. 27. 163-169.

Pennycuick. C. J. (1989) Bird Flight Performance. A Practical Calculation Guide. Oxford University Pess.

Pennycuick. C. J. (1991) Adapting skeletal muscles to be efficient. In: Efficiency, Economy and Related Concepts in Comparative Animal Physiology. (Edited by Blake. R. W.). Oxford Univ. Press.

Peters, S. E. (1989) Structure and function in vertebrate skeletal muscle. Amer. Zool. 29. 221-234.

Peterson. J. A., Benson, J. A.. Ngai, M. M., Morin, J. G. and Owe, C. (1982) Scaling in tensile "skeletons": Structures with scale-independent length dimensions. Science 217. 1267-1270.

Peterson, J. A., Benson, J. A., Morin, J. G. and McFall-Ngai, M. J. (1984) Scaling in tensile "skeletons": Scale dependent length of the Achilles tendon in mammals. J. Zool. 202(3), 361-372.

Prosser. C. L. (1958) Physiological Adaptation; A Symposium held during the Meeting of the Society of General Physiologists at the Marine Biological Laboratory. Woods Hole, Massachusetts. American Physiological Socicty. Washinglon, D.C.

Rall.J. A. (1985) Energetic aspects of skeletal muscle contraction: Implications of fiber types. Exerc. Sport Sci. Rev. 13, 33-74.
Riley. D. A., Ilyina-kakueva, E. I., Ellis, S., Bain, J. L. W., Slocum, G. R. and Sedlak, F. R. (1990) Skeletal muscle fiber, nerve, and blood vessel breakdown in space-flown rats. FASEB J. 4. 84-91.

Rome. L. C.. Funke, R. P., Alexander, R. M., Lutz, G., Aldridge, H.. Scoth, F. and Freadman, M. (1988) Why animals have different muscle fibre types. Nature (Lond) 335. 824-827.

Ruben. J. (1991) Reptilian physiology and the flight capacity of Archaeopteryx. Evol. 45(1). 1-17.

Schwarzacher. H. G. (1957)Zur Lage der motorischen Endplatten in den Skeletmuskein. Acta Anat. 30, 758-774.

Schwarzacher, H. G. (1959) Ueber die Lange und Anordnung der Muskelfasern der menschlischen Skeletmuskeln. Acta Anat. 37, 217-231.

Sutherland. S. (1952) The multiple motor innervation of individual muscle fibres in man. Acta Anat. 16. 167-168.

Swatland. H.J. and Cassens. R. G. (1972a) A brief study of muscle enlargement in the rat. J. Anim. Sci. 34, 21-24.

Swatland, H. J. and Cassens, R. G. (1972b) Muscle growth: The problem of muscle fibers with an intrafascicular termination. J. Anim. Sci. 35, 336-344.

Tardieu, C., Tabary, J. C., Tabary, C. (1981) Adaptation of sarcomere numbers to the length imposed on the muscle. Adv. Physiol. Sci. 24, 99-114.

Trotter. J. A. (1990) Interfiber tension transmission in seriesfibered muscles of the cat hindlimb. J. Morphol. 206. $351-361$.

Weeks. O. I. (1989) Vertebrate skeletal muscle: Power source for locumotion. Bio.Sci. 39, 791-799.

Wineski, L. E. and Herring. S. W. (1985) Innervation and function in the masseter complex of the pig. Fortschr. Zool. 30 , 285-287. 